\title{
Overview of gastroesophageal junction cancers
}

\author{
Aung Myint Oo, Saleem Ahmed \\ Department of General Surgery, Tan Tock Seng Hospital, Singapore 308433, Singapore. \\ Correspondence to: Dr. Saleem Ahmed, Department of General Surgery, Tan Tock Seng Hospital, Singapore 308433, \\ Singapore. E-mail: medicsaleem@gmail.com; Dr. Aung Myint Oo, Department of General Surgery, Tan Tock Seng Hospital, \\ Singapore 308433, Singapore. E-mail: myint_oo_aung@ttsh.com.sg
}

How to cite this article: Oo AM, Ahmed S. Overview of gastroesophageal junction cancers. Mini-invasive Surg 2019;3:13. http://dx.doi.org/10.20517/2574-1225.2019.02

Received: 14 Jan 2019 First Decision: 25 Mar 2019 Revised: 10 Apr 2019 Accepted: 17 Apr 2019 Published: 6 May 2019

Science Editor: Tetsu Fukunaga Copy Editor: Cai-Hong Wang Production Editor: Huan-Liang Wu

\begin{abstract}
Oesophageal and gastroesophageal junction (GEJ) malignancy is the fastest growing cancer in the Western population. This together with the deadly nature of the disease has attracted increased attention from doctors and researchers alike. The increasing incidence has been primarily attributed to the increase in rates of obesity that in turn causes increased gastroesophageal reflux disease leading to Barrett's oesophagus and eventually adenocarcinoma of the oesophagus especially at the GEJ. We discuss the epidemiology, risk factors and the management of GEJ tumours.
\end{abstract}

Keywords: Cardioesophageal, gastroesophageal, upper gastrointestinal, cancer, tumour

\section{INTRODUCTION}

Oesophageal and gastroesophageal junction (GEJ) malignancy is the fastest growing cancer in the Western population, especially in United States of America (USA), rising by 6-fold annually on the background of declining rates of most other cancers ${ }^{[1,2]}$. This together with the deadly nature of the disease has attracted increased attention from doctors and researchers alike. The increasing incidence has been primarily attributed to the increase in rates of obesity that in turn causes increased gastroesophageal reflux disease (GERD) leading to Barrett's oesophagus and eventually adenocarcinoma of the oesophagus especially at the $\mathrm{GEJ}^{[3]}$.

\section{DEFINITION}

The definition of GEJ cancers has been an area of controversy and disagreement and have in the past been considered either a gastric or oesophageal cancer as they lie in between the two. GEJ tumours also

cc (i) (c) The Author(s) 2019. Open Access This article is licensed under a Creative Commons Attribution 4.0 International License (https://creativecommons.org/licenses/by/4.0/), which permits unrestricted use, sharing, adaptation, distribution and reproduction in any medium or format, for any purpose, even commercially, as long as you give appropriate credit to the original author(s) and the source, provide a link to the Creative Commons license, and indicate if changes were made.

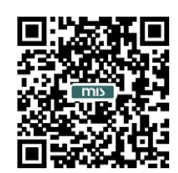


go by many other names including distal oesophageal cancers, proximal gastric cancers and cancers of cardia. This has led to discrepancies in the literature regarding the classification, pathophysiology, surgical approach and prognosis. The most widely accepted definition for GEJ cancer is that proposed by Siewert et al. ${ }^{[4]}$ and led to the tumours being classified as a distinct entity from gastric or oesophageal cancers. He proposed that GEJ tumours be classified as those having epicentre of the cancer within $5 \mathrm{~cm}$ proximal or distant to the Z-line. The Union for International Cancer Control (UICC) has adopted a similar definition and suggests that tumours that extend into the oesophagus classified and staged using an oesophageal scheme while those without oesophageal extension are staged using the gastric cancer scheme even if they occur within $5 \mathrm{~cm}$ distant to the GEJ ${ }^{[5]}$. American Joint Committee on Cancer (AJCC) suggests that cancers involving the GEJ that have their epicentre within the proximal $2 \mathrm{~cm}$ of the cardia (Siewert Type I/ II) are to be staged as oesophageal tumours while those that are more than $2 \mathrm{~cm}$ distal to the cardia should be staged as gastric cancer ${ }^{[6]}$.

The importance of accurate and reproducible definition of GEJ cancer is important as the tumour biology, management is different and importantly the prognosis is considered worse than that of oesophageal and gastric cancer. Even within GEJ tumours there is marked heterogeneity, Siewert Type II and III are known to have better prognosis than Siewert $\mathrm{I}^{[3]}$. GEJ tumours are known to demonstrate aggressive behaviour with early local invasion and systemic dissemination. As the GEJ tumours borders both the thoracic and abdominal cavities there is lymph outflow and therefore lymph node metastasis to both these regions. Moreover, the close relationship of the GEJ cancer to an anatomically complex region bordering multiple organs means complete resection can only be achieved by multi-visceral resection ${ }^{[7]}$.

\section{EPIDEMIOLOGY}

GEJ tumour incidence has dramatically increased in Western population and the distal oesophageal cancer type is the dominant oesophageal cancer type in the USA. Similarly, the rates of proximal gastric cancer have increased while that of distal gastric cancer has dropped. The rates of GEJ tumours has increased between $4 \%-10 \%$ every year in USA since $1976^{[8-10]}$. The overall proportion of proximal gastric cancer, cancer of the cardia and distal oesophageal cancer accounted for about 30\%-40\% of all gastric carcinoma in Western countries and interestingly, China, far higher than other Eastern centres such as Japan and Korea $^{[3,8,9]}$. However, these numbers have to be taken with a pinch of salt. Historically, the confusion in the labelling of GEJ tumours with them being previously labelled as oesophageal or gastric cancers or even "unspecified" under the World Health Organization's International Classification of Diseases for Oncology codes could lead to an erroneous result. A study conducted in Sweden revealed that the true incidence could be up to $45 \%$ higher or $15 \%$ lower than reported in its national registry ${ }^{[11]}$. The secular trend of rising incidence of GEJ tumours has to be interpreted with caution.

\section{RISK FACTORS}

The etiology of GEJ tumours is still unclear. Much of its alarming rise has been blamed on increasing trends of obesity and GERD. This is likely to explain the rise in Siewert I tumours, which arise from areas of intestinal metaplasia in the distal oesophagus contributed by chronic GERD due to obesity. However, it is still unclear if metaplasia is due to acid reflux or bile reflux into oesophagus. Analysis of oesophageal fluid in patients with GERD found that they contain 10 times more bile than normal controls ${ }^{[12]}$. Animal studies also have shown that duodenal fluid induces Barrett's oesophagus and oesophageal adenocarcinoma ${ }^{[13,14]}$. This might explain why use of acid suppression agents has not lowered the rates of GEJ tumours ${ }^{[1]}$.

Increasing rates of GERD due to acid or bile irritation does not explain the rise in Siewert Type II and III tumours. An analysis of GEJ tumours by Siewert et al. ${ }^{[15]}$ found that in contrast to Siewert Type I tumours 
intestinal metaplasia was only found in $10 \%$ of patients with Type II and rare in patients with Type III tumours which suggests chronic GERD cannot fully explain the surge in Type II and III tumours and that other factors maybe at play.

Obesity has been consistently implicated as a risk factor for development of GEJ. Apart from the mechanical pathway resulting in increased reflux, there maybe independent inflammatory and hormonal mediators. An Australian study, showed that obesity in combination with frequent reflux were risk factors for development of GEJ tumours than either acting alone suggesting that synergistic as opposed to additive effects was most likely ${ }^{[16]}$.

Infection with $H$. pylori is associated with increased rates of gastric cancer via mechanisms of chronic inflammation however there is an inverse association with $H$. pylori infection and incidence of GEJ tumours. A study by Whiteman et al. ${ }^{[17]}$ found that $H$. pylori was inversely associated with GEJ adenocarcinoma tumours with odds ratio (OR) 0.41. It is not well understood how the infection confers a protective benefit but the potential mechanism include decreased acid production and microbiome alteration $^{[18]}$. It is interesting note that the decreasing incidence of $H$. pylori over time parallels the increasing incidence of GEJ tumours.

Smoking has been found to have a strong association with GEJ tumours. A pooled analysis of multiple primary studies from the Barrett's Esophagus and Esophageal Adenocarcinoma Consortium (BEACON $)^{[19]}$ found that smoking was positively associated with GEJ adenocarcinoma ( $\mathrm{OR}=2.18,95 \% \mathrm{CI}$ : 1.84-2.58), and also demonstrated a strong dose-response association.

The other factors found to be associated with GEJ tumours include alcohol intake, intake of highly processed meat diet while intake of high fibre diet and medications such as non-steroidal anti-inflammatory drugs are thought to confer a protective effect ${ }^{[18]}$. Further studies are required to provide more conclusive evidence.

\section{MANAGEMENT}

Management of GEJ tumours is challenging as they involve two contiguous organs and also straddle the thoracic cavity and abdominal cavity via hiatal opening.

Management of GEJ cancers depends on the stage of the tumour. For early tumours there is a role for endoscopic submucosal dissection (ESD) but advanced tumours require multimodality treatment including surgery.

\section{Role of endoscopic treatment in early stage GEJ cancer}

With the development of the Insulated-tip diathermic knife (IT-knife) in late 1990 and subsequent development of the ESD technique in 2003, the endoscopic management of early gastrointestinal tumours including early gastric cancer and early esophageal cancer became feasible and popular ${ }^{[20-23]}$. ESD has been accepted as the minimally invasive curative treatment option for superficial early gastrointestinal cancers including those of stomach, esophagus and colonic origins ${ }^{[24]}$. However, the indication of endoscopic treatment for early GEJ cancers including Barret's adenocarcinoma has not been clearly established due to unclear pattern of lymph node metastasis ${ }^{[25]}$. Meta-analysis of 6 retrospective studies have demonstrated the safety and feasibility of ESD on early superficial GEJ cancers ${ }^{[26]}$. Five studies used curative criteria for gastric cancer and one study used the criteria for esophageal cancer. 269 patients who met the curative resection criteria did not have any local or distant metastases. Out of 90 patients who underwent noncurative resection, $3(3.3 \%)$ presented with local recurrence and $2(2.2 \%)$ presented with distant metastasis. The study was limited by the small number of patients with short duration of follow-up. A group from Korea compared outcomes of 79 patients with Siewert II adenocarcinoma who underwent ESD 
compared to surgery. The 5-year overall survival rates were similar in both groups. There was no gastric cancer related death in each group and the incidence of treatment-related adverse events was similar in both groups leading the authors to conclude that ESD may be an effective alternative to surgery with comparable long-term oncologic outcomes ${ }^{[27]}$. ESD has a good safety profile with low number of adverse events reported by many centers ${ }^{[28,29]}$. According to one comparative study, ESD was associated with much less adverse events compared to surgery group (10\%vs. 17.9\%). ESD is a highly skilled procedure mainly performed in Asian centers and many authors have described a steep learning curve for those performing ESD especially for GEJ tumours, therefore appropriate training including simulation based practice within a training framework must be enforced to increase safety and efficacy ${ }^{[30-32]}$. Based on current evidence, ESD for superficial early EGJ cancers is feasible and safe with favorable long-term outcomes however further work is necessary to establish specific resection criteria for ESD of GEJ tumours.

\section{Surgical management of GEJ tumours}

There are various surgical strategies for management of GEJ cancers. They include esophagectomy with partial gastrectomy or extended total gastrectomy with or without thoracotomy. Individualization of the surgical strategy and adherence to sound oncological principles with aims of radical lymphadenectomy with negative margins for the resectable GEJ tumours is key in attaining good outcomes. Tumour location as per Siewert Classification and location of enlarged lymph nodes are critical factors in determining the surgical strategy.

The pattern and frequency of lymph node metastasis differ according to the epicenter of the tumour location and histology ${ }^{[33-36]}$. Matsuda et al. ${ }^{[37]}$ carried out clinicopathological correlation of surgically resected GEJ tumours and found that the frequency of mediastinal lymph node metastasis was also found to be higher in squamous cell carcinoma than adenocarcinoma (46.7\% vs. $7.5 \%)$.

Regardless of the Siewert classification, the majority of the junctional tumours metastasise to the perigastric/abdominal regional lymph nodes ${ }^{[36,37]}$. The frequency of mediastinal lymph node metastasis differs significantly depending on the Siewert types. The mediastinal lymph node metastasis was only 9\% in Type III compared to $30 \%$ in Type II and $46 \%$ in Type I tumours while abdominal lymph nodes metastasis are found to be $51 \%, 71 \%$ and $91 \%$ respectively for Type I, II and III tumours ${ }^{[35]}$. Hence, choice of surgery and lymphadenectomy need to be tailored according to the epicentre and histology of the tumor.

Siewert type I tumours are considered as lower esophageal tumours with potential lymph node metastasis to mediastinal and abdominal lymph nodes. Subtotal esophagectomy with partial gastrectomy is considered to be a superior approach for type 1 tumours. Siewert type 3 tumours are considered proximal gastric cancer with potential lymph node metastasis to lower mediastinal and abdominal lymph nodes. Extended total gastrectomy with distal esophagectomy is considered more appropriate for type 3 tumour ${ }^{[15,38-40]}$.

Siewert type II tumours are true junctional tumours and choice of surgical approach is controversial. In a retrospective study comparing transmediastinal esophagectomy with partial gastrectomy and extended total gastrectomy with transhiatal distal esophagectomy, it was demonstrated that the latter was associated with fewer post-operative morbidity and mortality without any difference in survival ${ }^{[38]}$. However, the subgroup analysis of those patients with Ro resection showed that extended total gastrectomy and not a transmediastinal oesophagogastrectomy was an independent predictor for long term survival.

There were two phase III randomized control trials which compared two operative strategies for GEJ cancers ${ }^{[41,42]}$. In the Dutch Trial, 220 patients with Siewert Type I and II tumours were randomly assigned to transhiatal esophagectomy (THE) vs. extended transthoracic esophagectomy and en-bloc lymphadenectomy (via right thoracic cavity) (TTE) ${ }^{[41]}$. THE was associated with lower morbidity such as 
pulmonary complications and chylothorax. Although median overall, disease-free, and quality-adjusted survival did not differ statistically between the groups, there was a trend toward improved long-term survival at five years with the extended transthoracic approach. In the follow-up study on 5 year survival, there was no significant overall survival benefit for either approach (36\% in TTE vs. $34 \%$ in THE, $P=$ $0.71^{[43]}$. However, extended TTE for type I esophageal adenocarcinoma showed a trend towards better 5 -year survival $(51 \%$ vs. $37 \%, P=0.33)$. Moreover, patients with a limited number of positive lymph nodes in the resection specimen seem to benefit from an extended transthoracic esophagectomy.

In the Japanese JCOG 9502 trial, patients with Siewert II and III cancers were assigned to either transhiatal approach (TH) or left thoracoabdominal approach (LTA $)^{[42]}$. TH consisted of a total gastrectomy with D2 lymphadenectomy (including splenectomy) via a laparotomy. Thoracotomy on either side was allowed to achieve a complete (Ro) resection only when the proximal surgical margin was positive (determined either macroscopically or microscopically by frozen section) and no further transhiatal oesophageal resection was possible. In LTA group, a thorough mediastinal nodal dissection below the left inferior pulmonary vein was undertaken with oesophagectomy of sufficient length. The trial was closed prematurely as the planned interim analysis concluded that the LTA approach was associated with higher morbidity and mortality including postoperative complications such as pulmonary complications; $49 \% v s .34 \%, P=0.06$ ) and in-hospital mortality ( $4 \% v$ s. $0 \%, P=0.25)$. Although statistically insignificant, both 5 years $(38 \% v s .52 \%)$ and 10 years survival $(24 \%$ vs. $37 \%)$ were found to be lower with LTA group ${ }^{[44]}$.

Complete Ro resection of GEJ tumours is key to achieving good survival outcomes. Systematic review and meta-analysis of fourteen studies involving 2433 patients with oesophageal cancer who had undergone oesophagectomy showed that circumferential resection margin (CRM) involvement was associated with significantly higher 5-year mortality rate (OR 2.05, 95\%CI: 1.41-2.99; $P<0.001)^{[45]}$.

There are few options for reconstruction after resection of GEJ tumours. For Type II and Type III tumours, after extended total gastrectomy and distal esophagectomy the reconstruction is usually done with Roux-En-Y esophago-jejunostomy. For Type I tumours, combined transabdominal and transthroaic approach is required to perform enbloc eosophagectomy and proximal gastrectomy together with 2 field lymphadenectomy. The other available option is a left throacoabdominal approach with intrathoracic anastomosis know as Sweet esophagectomy. The stomach is used as a conduit to perform intrathoracic oesophagogastrostomy for reconstruction. In some patients, if gastric conduit is unsuitable, due to previous surgery, the jejunum and colon are both possible conduit options.

\section{Open versus minimally invasive approach}

Evidence suggests that transthoracic approach with radical lymphadenectomy may be an oncologically superior operation with better long-term survival with the downsides of increased operative morbidity and mortality especially pulmonary complications. Minimally invasive approaches may be a promising alternative with decreased post-operative complications without compromising the radicality of the surgery. The current evidence suggests the potential benefits for minimally invasive esophagectomy approach to GEJ tumours include smaller incisions, less intraoperative blood loss fewer postoperative complications, shorter admission to the intensive care unit and overall hospital stay, better preservation of postoperative pulmonary function and equivalent quality of lymph node dissections ${ }^{[46-49]}$. The evidence for minimally invasive surgeries for GEJ tumours is convincing, however more prospective studies are required to evaluate the long-term oncological outcomes.

\section{Multimodality management of GEJ tumours}

Multimodality treatment strategies in locally advanced GEJ tumours (T2 and higher or node positive) result in improved outcomes. These strategies include neoadjuvant and adjuvant chemotherapy with 
or without radiation therapy in addition to surgery. The multimodality treatment has now become the standard of care for advanced GEJ cancers. However, the best approach to multimodality treatment for GEJ cancers is not established yet as GEJ tumours represent only a small subset of cohort in most of clinical trials ${ }^{[50-53]}$. Even though adjuvant chemotherapy has been proven to be beneficial and improve survival outcomes compared to surgery alone in gastric cancers, the role of adjuvant chemotherapy in GEJ tumours is still unclear as there are no large trials conducted for GEJ cancers specifically and are often categorised under the subset of gastric cancers ${ }^{[54,55]}$.

Adjuvant chemoradiotherapy is one possible option for patients with GEJ tumours who didn't receive the preoperative treatment with survival benefit demonstrated in US intergroup 0116 trial $^{[53]}$. This regimen also known as the "Macdonald regimen" is the current standard of adjuvant treatment for patients with gastric cancer. Of the 559 patients recruited in this trial only 20\% had GEJ cancers. Adjuvant chemoradiotherapy produced substantial reduction in both overall relapse and locoregional relapse as well improved overall survival, hazard ratio (HR) $1.32(95 \% \mathrm{CI} \text { : } 1.10-1.60 ; P=0.0046)^{[53]}$. One major criticism of the study was that only $10 \%$ of patients had received D2 lymphadenectomy and therefore the improvement in relapse rates and survival could be due to potential compensation of an oncologically inadequate surgery.

Neoadjuvant or perioperative chemotherapy with or without radiation therapy have proven to be effective in improving survival. The MAGIC trial compared patients who underwent surgery alone to surgery plus perioperative chemotherapy ( 3 cycles of preoperative and 3 cycles of postoperative epirubicin, cisplatin and infusional flurouracil $)^{[50]}$. Of the 503 patients recruited only $11 \%$ had GEJ tumours. Compared to surgery alone group, the perioperative chemotherapy group had higher overall survival (HR for death, 0.75; 95\%CI: $0.60-0.93 ; P=0.00936$ percent vs. 23 percent) and progression-free survival (HR for progression, 0.66 ; 95\%CI: 0.53-0.81; $P<0.001)$.

The Dutch Chemoradiotherapy for Esophageal Cancer Followed by Surgery Study (CROSS) trial included $22 \%$ of patients with GEJ cancers and compared the effectiveness of neoadjuvant chemoradiation over surgery alone ${ }^{[52]}$. Overall survival was significantly better in the neoadjuvant chemoradiotherapy group (HR, 0.657; 95\%CI: $0.495-0.871 ; P=0.003$ ). The survival benefit in chemoradiation group was persistent in the long term with median follow up of 86.4 months ${ }^{[56]}$.

The Preoperative therapy in Esophagogastric adenocarcinoma Trial (POET) was the only trial which compared neoadjuvant chemotherapy versus chemoradiotherapy in locally advanced GEJ adenocarcinoma ${ }^{[57]}$. Although the study ended prematurely due to lower accrual, there was a trend observed towards improved 3-year survival in the chemoradiation group ( $47.4 \%$ vs. $27.7 \%$; $P=0.07)$. The long-term follow-up of the patients also showed a trend in improved overall 5-year survival in favor of preoperative chemoradiotherarpy (HR 0.65, 95\%CI: 0.42-1.01, $P=0.055)$.

A meta-analysis including 24 studies concluded that there was strong evidence for survival benefit of neoadjuvant chemoradiotherapy or chemotherapy over surgery alone in patients with esophageal carcinoma including GEJ cancers $^{[58]}$. However, there was no clear advantage of neoadjuvant chemoradiotherapy over neoadjuvant chemotherapy according to the study.

\section{CONCLUSION}

The management of GEJ junction tumours is challenging and there is no one-size-fit-all strategy. The endoscopic option can be considered for early tumours especially for those patients with high risk for surgery. The surgical approach for advanced GEJ cancers should be tailored according to the histological subtype, extent of oesophageal and/or gastric invasion, clinical and radiological lymph node involvement, achievement of negative resection margins with Ro resection as well as achievement of safe anastomosis for 
reconstruction. Minimally invasive approach is promising especially in experienced hands however more data on long-term oncological results is needed.

Multimodality treatment is superior to surgery alone in locally advanced resectable GEJ tumours. The advantages of neoadjuvant or perioperative treatment are downstaging of tumour, reducing the risk of recurrence, improving rates of Ro resection and improving outcomes after complete resection. At present, there is no global consensus on the optimal multimodality management of GEJ tumours. Further studies are needed to explore the optimal treatment strategy including surgical approach, sequence and regimen of multimodality management .

\section{DECLARATIONS}

\section{Authors' contributions}

Oo AM and Ahmed S contributed equally to literature search and writing of manuscript.

\section{Availability of data and materials}

Not applicable.

\section{Financial support and sponsorship}

None.

\section{Conflicts of interest}

The authors declared that there are no conflicts of interest.

\section{Ethical approval and consent to participate}

Not applicable.

\section{Consent for publication}

Not applicable.

\section{Copyright}

(c) The Author(s) 2019.

\section{REFERENCES}

1. Chai J, Jamal MM. Esophageal malignancy: a growing concern. World J Gastroenterol 2012;18:6521-6.

2. Lerut T. Carcinoma of the esophagus and gastro-esophageal junction. 2001.

3. Wu AW, Ji JF. Adenocarcinoma of esophagogastric junction requires a clearer definition. Transl Gastrointest Cancer 2013;2:5-9.

4. Siewert JR, Stein HJ. Classification of adenocarcinoma of the oesophagogastric junction. Br J Surg 1998;85:1457-9.

5. Wittekind Ch, Sobin LH, Gospodarowicz MK. TNM classification of malignant tumours. 7th ed. Blackwell Publishing Ltd; 2009.

6. Amin MB, Edge S, Greene F, Byrd DR, Brookland RK, et al. AJCC Cancer Staging Manual. 8th ed. Springer; 2017.

7. Kshivets O. Cardioesophageal cancer: best treatment strategies. Open Cardiovasc Thorac Surg J 2009;2:21-32.

8. Strong VE, Song KY, Park CH, Jacks LM, Gonen M, et al. Comparison of gastric cancer survival following R0 resection in the United States and Korea using an internationally validated nomogram. Ann Surg 2010;251:640-6.

9. Wu AW, Ji JF, Yang H, Li YN, Li SX, et al. Long-term outcome of a large series of gastric cancer patients in China, Chinese J Cancer Res 2010;22:167-75.

10. Cellini F, Morganti AG, Di Matteo FM, Mattiucci GC, Valentini V. Clinical management of gastroesophageal junction tumors: past and recent evidences for the role of radiotherapy in the multidisciplinary approach. Radiat Oncol 2014;9:45.

11. Ekström AM, Signorello LB, Hansson LE, Bergström R, Lindgren A, et al. Evaluating gastric cancer misclassification: a potential explanation for the rise in cardia cancer incidence. J Natl Cancer Inst 1999;91:786-90.

12. Kauer WK, Peters JH, DeMeester TR, Feussner H, Ireland AP, et al. Composition and concentration of bile acid reflux into the esophagus of patients with gastroesophageal reflux disease. Surgery 1997;122:874-81.

13. Miwa K, Sahara H, Segawa M, Kinami S, Sato T, et al. Reflux of duodenal or gastro-duodenal contents induces esophageal carcinoma in rats. Int J Cancer 1996;67:269-74.

14. T. Miyashita, T. Ohta, T. Fujimura, I. Ninomiya, S. Fushida, et al. Duodenal juice stimulates oesophageal stem cells to induce Barrett's oesophagus and oesophageal adenocarcinoma in rats. Oncol Rep 2006;1469-75. 
15. Siewert JR, Feith M, Werner M, Stein HJ. Adenocarcinoma of the esophagogastric junction: results of surgical therapy based on anatomical/topographic classification in 1,002 consecutive patients. Ann Surg 2000;232:353-61.

16. Whiteman DC, Sadeghi S, Pandeya N, Smithers BM, Gotley DC, et al. Combined effects of obesity, acid reflux and smoking on the risk of adenocarcinomas of the oesophagus. Gut 2008;57:173-80.

17. Whiteman DC, Parmar P, Fahey P, Moore SP, Stark M, et al. Association of helicobacter pylori infection with reduced risk for esophageal cancer is independent of environmental and genetic modifiers. Gastroenterology 2010;139:73-83.

18. Buas MF, Vaughan TL. Epidemiology and risk factors for gastroesophageal junction tumors: understanding the rising incidence of this disease. Semin Radiat Oncol 2013;23:3-9.

19. Cook MB, Kamangar F, Whiteman DC, Freedman ND, Gammon MD, Bernstein L, et al. Cigarette smoking and adenocarcinomas of the esophagus and esophagogastric junction: a pooled analysis from the international BEACON consortium. J Natl Cancer Inst 2010;102:1344-53.

20. Fukuzawa M, Gotoda T. History of endoscopic submucosal dissection and role for colorectal endoscopic submucosal dissection: a Japanese perspective. Gastrointest Interv 2012;1:30-5.

21. Hosokawa K, Yoshida S. Recent advances in endoscopic mucosal resection for early gastric cancer. Gan To Kagaku Ryoho 1998;25:47683.

22. Ono H, Kondo H, Gotoda T, Shirao K, Yamaguchi H, et al. Endoscopic mucosal resection for treatment of early gastric cancer. Gut 2001:48:225-9.

23. Gotoda T, Kondo H, Ono H, Saito Y, Yamaguchi H, et al. A new endoscopic mucosal resection procedure using an insulation-tipped electrosurgical knife for rectal flat lesions: report of two cases. Gastrointest Endosc 1999;560-3.

24. Toyonaga T, Man-i M, East JE, Nishino E, Ono W, et al. 1,635 Endoscopic submucosal dissection cases in the esophagus, stomach, and colorectum: complication rates and long-term outcomes. Surg Endosc 2013;1000-8.

25. Nagami Y, Ominami M, Otani K, Hosomi S, Tanaka F, et al. Endoscopic submucosal dissection for adenocarcinomas of the esophagogastric junction. Digestion 2018;38-44.

26. Park CH, Kim EH, Kim HY, Roh YH, Lee YC. Clinical outcomes of endoscopic submucosal dissection for early stage esophagogastric junction cancer: a systematic review and meta-analysis. Dig Liver Dis 2015;47:37-44.

27. Gong EJ, Kim DH, Ahn JY, Jung KW, Lee JH, Choi KD, et al. Comparison of long-term outcomes of endoscopic submucosal dissection and surgery for esophagogastric junction adenocarcinoma. Gastric Cancer 2017;20:84-91.

28. Osumi H, Fujisaki J, Omae M, Shimizu T, Yoshio T, et al. Clinicopathological features of Siewert type II adenocarcinoma: comparison of gastric cardia adenocarcinoma and Barrett's esophageal adenocarcinoma following endoscopic submucosal dissection, Gastric Cancer 2017;20:663-70

29. Nagami Y, Machida H, Shiba M, Obayashi T, Ominami M, et al. Clinical efficacy of endoscopic submucosal dissection for adenocarcinomas of the esophagogastric junction. Endosc Int Open 2014;2:E15-E20.

30. Tanimoto MA, Villalobos GT, Fujita R, Doherty PS, Saavedra JA, et al. Learning curve in a western training center of the circumferential en bloc esophageal endoscopic submucosal dissection in an in vivo animal model. Diagn Ther Endosc 2011;2011:847831.

31. Oda I, Odagaki T, Suzuki H, Nonaka S, Yoshinaga S. Learning curve for endoscopic submucosal dissection of early gastric cancer based on trainee experience. Dig Endosc 2012;24 Suppl 1:129-32.

32. Kato M, Gromski M, Jung Y, Chuttani R, Matthes K. The learning curve for endoscopic submucosal dissection in an established experimental setting. Surg Endosc 2013;27:154-61.

33. Dresner SM, Lamb PJ, Bennett MK, Hayes N, Griffin SM. The pattern of metastatic lymph node dissemination from adenocarcinoma of the esophagogastric junction. Surgery 2001;129:103-9.

34. Kakeji Y, Yamamoto M, Ito S, Sugiyama M, Egashira A, et al. Lymph node metastasis from cancer of the esophagogastric junction, and determination of the appropriate nodal dissection. Surg Today 2012;42:351-8.

35. Pedrazzani C, De Manzoni G, Marrelli D, Giacopuzzi S, Corso G, et al. Lymph node involvement in advanced gastroesophageal junction adenocarcinoma. J Thorac Cardiovasc Surg 2007;134:378-85.

36. Siewert JR, Stein HJ, Feith M. Adenocarcinoma of the Esophago-Gastric Junction. Scand J Surg 2006;95:260-9.

37. Matsuda T, Takeuchi H, Tsuwano S, Nakamura R, Takahashi T, et al. Optimal surgical management for esophagogastric junction carcinoma. Gen Thorac Cardiovasc Surg 2014;62:560-6.

38. Feith M, Stein HJ, Siewert JR. Adenocarcinoma of the esophagogastric junction: surgical therapy based on 1602 consecutive resected patients. Surg Oncol Clin N Am 2006;15:751-64.

39. von Rahden BH, Stein HJ, Siewert JR. Surgical management of esophagogastric junction tumors. World J Gastroenterol 2006;12:6608-13.

40. Gertler R, Stein HJ, Langer R, Nettelmann M, Schuster T, et al. Long-term outcome of 2920 patients with cancers of the esophagus and esophagogastric junction. Ann Surg 2011;253:689-98.

41. Hulscher JB, van Sandick JW, A.G. de Boer, B.P. Wijnhoven, J.G.Tijssen, et al. Extended transthoracic resection compared with limited transhiatal resection for adenocarcinoma of the esophagus. N Engl J Med 2002;347:1662-9.

42. Sasako M, Sano T, Yamamoto S, Sairenji M, Arai K, et al. Left thoracoabdominal approach versus abdominal-transhiatal approach for gastric cancer of the cardia or subcardia: a randomised controlled trial. Lancet Oncol 2006;7:644-51.

43. Omloo JM, Lagarde SM, Hulscher JB, Reitsma JB, Fockens P, et al. Extended transthoracic resection compared with limited transhiatal resection for adenocarcinoma of the mid/distal esophagus. Ann Surg 2007;246:992-1000, discussion 1000-1.

44. Kurokawa Y, Sasako M, Sano T, Yoshikawa T, Iwasaki Y, et al. Ten-year follow-up results of a randomized clinical trial comparing left thoracoabdominal and abdominal transhiatal approaches to total gastrectomy for adenocarcinoma of the oesophagogastric junction or gastric cardia. Br J Surg 2015;102:341-8.

45. Chan DSY, Reid TD, Howell I, Lewis WG. Systematic review and meta-analysis of the influence of circumferential resection margin involvement on survival in patients with operable oesophageal cancer, Br J Surg 2013:100:456-64. 
46. Biere SS, van Berge Henegouwen MI, Maas KW, Bonavina L, Rosman C, et al. Minimally invasive versus open oesophagectomy for patients with oesophageal cancer: a multicentre, open-label, randomised controlled trial. Lancet 2012;379:1887-92.

47. Biere SSAY, Cuesta MA, van der Peet DL. Minimally invasive versus open esophagectomy for cancer: a systematic review and metaanalysis. Minerva Chir 2009;121-33.

48. Verhage RJJ, Hazebroek EJ, Boone J, Van Hillegersberg R. Minimally invasive surgery compared to open procedures in esophagectomy for cancer: a systematic review of the literature. Minerva Chir 2009;64:135-46.

49. Santillan AA, Farma JM, Meredith KL, Shah NR, Kelley ST. Minimally invasive surgery for esophageal cancer. J Natl Compr Canc Netw 2008:879-84.

50. Cunningham D, Allum WH, Stenning SP, Thompson GN, Van de Velde CJ, et al. Perioperative chemotherapy versus surgery alone for resectable gastroesophageal cancer. N Engl J Med 2006;355:11-20.

51. Medical Research Council Oesophageal Cancer Working Group. Surgical resection with or without preoperative chemotherapy in oesophageal cancer: a randomised controlled trial. Lancet 2002;359:1727-33.

52. van Hagen P, Hulshof MC, van Lanschot JJ, Steyerberg EW, van Berge Henegouwen MI, et al. Preoperative chemoradiotherapy for esophageal or junctional cancer. N Engl J Med 2012;366:2074-84.

53. Macdonald JS, Smalley SR, Benedetti J, Hundahl SA, Estes NC, et al. Chemoradiotherapy after surgery compared with surgery alone for adenocarcinoma of the stomach or gastroesophageal junction. N Engl J Med 2001;345:725-30.

54. Bang YJ, Kim YW, Yang HK, Chung HC, Park YK, et al. Adjuvant capecitabine and oxaliplatin for gastric cancer after D2 gastrectomy (CLASSIC): a phase 3 open-label, randomised controlled trial. Lancet 2012;379:315-21.

55. Sakuramoto S, Sasako M, Yamaguchi T, Kinoshita T, Fujii M, et al. Adjuvant chemotherapy for gastric cancer with S-1, an oral fluoropyrimidine. N Engl J Med 2007;357:1810-20.

56. Shapiro J, van Lanschot JJB, Hulshof MCCM, van Hagen P, van Berge Henegouwen MI, et al. Neoadjuvant chemoradiotherapy plus surgery versus surgery alone for oesophageal or junctional cancer (CROSS): long-term results of a randomised controlled trial. Lancet Oncol 2015;16:1090-8.

57. Stahl M, Walz MK, Stuschke M, Lehmann N, Meyer HJ, et al. Phase III comparison of preoperative chemotherapy compared with chemoradiotherapy in patients with locally advanced adenocarcinoma of the esophagogastric junction. J Clin Oncol 2009;27:851-6.

58. Sjoquist KM, Burmeister BH, Smithers BM, Zalcberg JR, Simes RJ, et al. Survival after neoadjuvant chemotherapy or chemoradiotherapy for resectable oesophageal carcinoma: an updated meta-analysis. Lancet Oncol 2011;12:681-92. 